\title{
Effect of fibroblasts on breast cancer cell mammosphere formation and regulation of stem cell-related gene expression
}

\author{
FENGCHUN ZHANG ${ }^{1}$, CAILI SONG ${ }^{1}$, YUE MA $^{2}$, LEI TANG $^{2}$, YINGCHUN XU $^{2}$ and HONGXIA WANG ${ }^{2}$ \\ ${ }^{1}$ Department of Oncology, Suzhou Kowloon Hospital, Shanghai Jiaotong University School of Medicine, \\ Suzhou 215021; ${ }^{2}$ Department of Oncology, Shanghai Renji Hospital, \\ Shanghai Jiaotong University School of Medicine, Shanghai 200127, P.R. China
}

Received February 24, 2011; Accepted April 20, 2011

DOI: $10.3892 / \mathrm{ijmm} .2011 .700$

\begin{abstract}
The purpose of this study was to investigate the regulatory effects of breast cancer fibroblasts (BCFs) vs. normal mammary fibroblasts (NMFs) on mammosphere formation and stem cell-related gene expression in breast cancer cells. Breast cancer cells (MCF-7) were cultured in suspension to generate primary and secondary mammospheres. The proportion of $\mathrm{CD} 44^{+} / \mathrm{CD} 24^{\text {low } /}$ cells was assessed by flow cytometry (FCM), and Wnt1, Notch1, $\beta$-catenin, CXCR4, SOX 2 and $A L D H 3 A 1$ gene expression was detected by quantitative realtime PCR. The fibroblasts from either breast cancer tissue or normal mammary tissue were purified from tissue specimens and co-cultured with breast cancer cells. The mammosphere formation efficacy was approximately 180/10,000 MCF-7 cells. FCM analysis showed that, compared to the $2.1 \%$ positive expression in the MCF-7 monolayer culture cells, the expression of $\mathrm{CD} 44^{+} / \mathrm{CD} 24^{\text {low/ }}$ in MCF-7 mammosphere cells was significantly elevated to $10.4 \%(\mathrm{P}<0.01)$. The proportion of the $\mathrm{CD} 44^{+} / \mathrm{CD} 24^{\text {low/ }}$ subpopulation of the cells in mammospheres was nearly 5-fold higher than that of general MCF-7 cells. Compared with MCF-7 monolayer culture cells, mammosphere cells showed significantly $(\mathrm{P}<0.01)$ enhanced expression of Wntl [fold-change (FC), 2.25], Notchl (FC, 2.45), $\beta$-catenin (FC, 1.72), CXCR4 (FC, 4.68), SOX2 (FC, 4.25) and ALDH3A1 (FC, 5.38). When BCFs were co-cultured with MCF-7 cells under mammosphere culture conditions, the length of time of mammosphere formation decreased, the volume of the mammospheres increased and the mammosphere-forming efficiency (MFE) was higher than that of NMFs and the control group. Both the BCF and NMF groups showed enhanced gene expression for the following genes: Wntl (FC, 3.18 and 1.27, respectively), $\beta$-catenin (FC, 1.75 and 1.22 , respectively), Notchl (FC, 2.09 and 1.31, respectively), CXCR4 (FC, 2.77 and
\end{abstract}

Correspondence to: Dr Hongxia Wang or Dr Yingchun Xu, Department of Oncology, Shanghai Renji Hospital, Shanghai Jiaotong University School of Medicine, Shanghai 200127, P.R. China

E-mail:whx365@126.com

E-mail: xiaoxu2384@163.com

Key words: breast cancer, stem cell, mammosphere, fibroblast cells
1.33 , respectively), $S O X 2$ (FC, 2.77 and 1.80, respectively) and ALDH3Al (FC, 5.23 and 1.85, respectively). Cancer fibroblast cells can promote the MFE and up-regulate stem cell-related gene expression in breast cancer cells.

\section{Introduction}

According to the cancer stem cell (CSC) theory, a small population of tumor cells is capable of self-renewal, which gives rise to the heterogeneous nature of tumors. CSCs have been discovered in multiple cancers, including those of the hematopoietic system, brain, breast, prostate, and gastrointestinal tract (1-4). These cells can exclude dyes such as Hoechst or rhodamine due to the increased expression of membrane transporter proteins, including p-glycoproteins or breast cancer resistance proteins (BCRPs) (5). Therefore, it is difficult for conventional therapies to eradicate CSCs, which largely contributes to patient relapse, years after chemotherapy or radiotherapy.

In 2003, Clarke and his colleagues identified human breast cancer stem cells by their CD $44^{+} / \mathrm{CD} 24^{-}$phenotype. These rare cell populations possessed an increased ability to form tumors when they were injected into etoposide-treated NOD/ SCID mice; as few as $100 \mathrm{CD} 44^{+} / \mathrm{CD} 24^{-}$human breast cancer cells could recapitulate the human mammary tumors, whereas the injection of 10,000 cells with other phenotypes failed to give rise to tumors (2). Since then, several techniques have been established to isolate or enrich for tumorigenic breast cancer stem cells (BCSCs), including side-population (SP) separation and mammosphere culture $(6,7)$. In breast cancer, the mammosphere culture system has been widely used to identify and enrich for putative stem cells using breast cancer cell lines (8). In a previous study, we described an in vitro culture system to propagate primary human mammary epithelial stem cells and progenitor cells in an undifferentiated state based on their ability to proliferate in suspension as spherical structures when cultured on non-adherent surfaces in the presence of growth factors. We found that cultured mammospheres exhibited the characteristics of BCSCs using this method (9).

In some reports, mammary stem/progenitor cells have been described as small, light cells that lie in an intermediate location, termed a stem cell niche (10) that is composed of stromal cells (myofibroblasts, endothelial cells and inflammatory 
cells) and extracellular matrix (ECM) components (e.g., laminin, fibronectin, collagen, and proteoglycans), which may influence mammary development. BCSCs also have a functional niche; stromal cells, together with ECM components, provide the microenvironment that is pivotal for BCSC self-renewal, proliferation, and maintenance in an undifferentiated state. Based on this finding, recent studies have mainly focused on how to regulate mammospheres. Fibroblasts are considered to play a central role in the complex process of tumor-stromal interactions and consequently also in tumorigenesis. Premalignant mammary epithelial cells exposed to senescent human fibroblasts irreversibly become invasive and undergo full malignant transformation. In invasive breast cancer, fibroblasts were found in a much higher proportion than in situ carcinomas and were predominantly at the invasive front.

However, the regulatory effects of fibroblasts on mammosphere formation remain unclear. We performed this study to further investigate the interactive influence of fibroblasts on BCSCs. The aim of the study was to compare the effect of breast cancer fibroblasts (BCFs) and normal mammary fibroblasts (NMFs) on the MCF-7 breast cancer cell mammosphere formation and signaling pathway regulation, which will help us to understand the interaction between the microenvironment and breast cancer stem cells.

\section{Materials and methods}

Cell culture and reagents. MCF-7 human breast epithelial adenocarcinoma cells were obtained from the American Type Culture Collection (ATCC, Manassas, VA). Cells were cultured routinely in Dulbecco's modified Eagle's medium/F12 (DMEM/F12, Hyclone, Logan, UT) supplemented with $10 \%$ fetal bovine serum (FBS), $100 \mathrm{U} / \mathrm{ml}$ penicillin and $100 \mu \mathrm{g} / \mathrm{ml}$ streptomycin (Life Technologies, Inc., Grand Island, NY) at $37^{\circ} \mathrm{C}$ in a humidified atmosphere with $5 \% \mathrm{CO}_{2}$.

Epidermal growth factor (EGF), insulin, basic fibroblast growth factor (bFGF), B27, TRIzol and primers for PCR were purchased from Invitrogen (Carlsbad, CA). Moloney murine leukemia virus (MMLV) reverse transcriptase was purchased from Promega (Madison, WI). Heparin, type I collagenase and hyaluronidase were obtained from Sigma (St. Louis, MO). Fibroblast-specific protein (FSP) antibody was purchased from NeoMarkers (Pittsburgh, PA).

Mammosphere culture. To obtain BCSCs and propagate them as mammospheres, cells floating in the supernatant of 2-dayold cultures were collected by centrifugation, washed in Hank's-buffered salt solution, and plated in ultralow attachment plates (Corning, NY) at a density of $2 \times 10^{4}$ viable cells $/ \mathrm{ml}$. The cells were then grown in serum-free mammary epithelial growth medium (BioWhittaker, Walkersville, MD) supplemented with B27, $10 \mathrm{ng} / \mathrm{ml} \mathrm{EGF,} 5 \mu \mathrm{g} / \mathrm{ml}$ bovine insulin, $20 \mathrm{ng} / \mathrm{ml}$ bFGF2 and $4 \mu \mathrm{g} / \mathrm{ml}$ heparin. Bovine pituitary extract was excluded from the growth medium. Growth factors were added to the mammosphere cultures every 3 days, and mammospheres ( $>60 \mu \mathrm{M}$ in size) were counted on Day 7 .

By Day 7, non-adherent spherical clusters of cells were harvested by trypsinization and centrifugation and dispersed by passage through a $40-\mu \mathrm{m}$ pore filter. The single cells were then collected for further analysis.
Flow cytometry. Mammospheres were dispersed to obtain single-cell populations as described above. Cells were washed in phosphate-buffer saline (PBS) with $2.5 \%$ bovine serum albumin and stained with FITC-anti-mouse CD24 and APC-anti-mouse CD44 antibodies (BD Pharmingen, San Jose, CA). Cells were incubated on ice for $30 \mathrm{~min}$, washed twice with PBS and then fixed in PBS containing paraformaldehyde. Flow cytometric analysis was performed on a BD FACSCalibur system (BD Biosciences, San Jose, CA), and acquisition was performed with the BD CellQuest software (BD Biosciences). The FlowJo software (Tree Star, Ashland, OR) was used for data analysis.

Isolation and culture of fibroblasts. Tumor specimens were obtained after obtaining informed consent from the patients and receiving approval from the Ethics Committee. Malignant $(n=5)$ and normal mammary tissue samples (from the same patient but from a site remote of the tumor area and free from tumor cells, as confirmed by subsequent histological analysis; $\mathrm{n}=5$ ), without neoadjuvant chemotherapy were collected immediately after surgery and mechanically disaggregated under sterile conditions within $30 \mathrm{~min}$.

The procedure was performed as previously described, with slight modifications. In brief, fresh tumor tissues were dissected to a volume of $1 \mathrm{~mm}^{3}$, with the elimination of fat, and subjected to $100 \mathrm{U} / \mathrm{ml}$ type 1 collagenase and $150 \mathrm{U} / \mathrm{ml}$ hyaluronidase treatment under constant stirring at $37^{\circ} \mathrm{C}$ for $18 \mathrm{~h}$. The resulting cell suspension was passed through an $80-\mu \mathrm{m}$ filter, and the single cell suspension was centrifuged at $80 \mathrm{x}$ g for $6 \mathrm{~min}$ and seeded in $25-$ or $75-\mathrm{cm}^{2}$ tissue culture flask. Cultures were fed twice a week, and after 7 days, when $60-80 \%$ confluence had been reached, stromal fibroblasts were obtained by differential trypsinization. The resulting second-passage fibroblasts were then seeded into a separate flask and maintained in DMEM/F12 supplemented with $10 \%$ FBS, $100 \mathrm{U} / \mathrm{ml}$ penicillin, and $100 \mathrm{U} / \mathrm{ml}$ streptomycin in a $37^{\circ} \mathrm{C}$ incubator containing $5 \% \mathrm{CO}_{2}$. Cells were maintained in culture for up to 2-4 passages.

Immunocytochemistry. The suspension of fibroblasts obtained was cultured on sterile glass coverslips for $24 \mathrm{~h}$, after which the coverslips were rinsed with PBS and the cells were fixed with $4 \%$ (vol/vol) acetic acid in methanol. The sections were treated with $3 \%$ hydrogen peroxide in methanol for $30 \mathrm{~min}$ and then $10 \%$ BSA for $30 \mathrm{~min}$ at room temperature. Primary antibodies against vimentin, $\alpha$-SMA, and FSP were added separately, and the samples were incubated at $37^{\circ} \mathrm{C}$ for $24 \mathrm{~h}$. After washing with PBS, a secondary antibody against the rabbit antibody (diluted 1:200 in PBS) was added for a $30 \mathrm{~min}$ incubation at $37^{\circ} \mathrm{C}$, and the immunoreactivity was visualized by immersing the sample in a diaminobenzidine (DAB)- $\mathrm{H}_{2} \mathrm{O}_{2}$ solution (0.05 M Tris- $\mathrm{HCl}$ buffer, $\mathrm{pH} 7.6$, with $0.05 \%$ DAB and $0.01 \% \mathrm{H}_{2} \mathrm{O}_{2}$ ) for $5 \mathrm{~min}$ at room temperature. The sections were finally stained with hematoxylin and mounted for light microscopy analysis.

Transwell assay. Mammospheres were co-cultured with either NMFs or BCFs using a transwell system. Fibroblasts $\left(2 \times 10^{5}\right.$ cells in $2 \mathrm{ml} /$ well) were seeded into the upper chamber of the transwell, and mammospheres $\left(2 \times 10^{5}\right.$ cells $\left./ \mathrm{ml}\right)$ were cultured in 
Table I. Primers used in real-time PCR analysis.

\begin{tabular}{|c|c|c|}
\hline Gene & Primer sequence & Product size (bp) \\
\hline$G A P D H$ & $\begin{array}{l}\text { (F) 5'-ACCCACTCCTCCACCTTTGA-3' } \\
\text { (R) 5'-CTGTTGCTGTAGCCAAATTCGT-3' }\end{array}$ & 101 \\
\hline Wntl & $\begin{array}{l}\text { (F) 5'-GAACCTGCTTACAGACTCCAAGAGT-3' } \\
\text { (R) 5'-CCGGATTTTGGCGTATCAGA-3' }\end{array}$ & 98 \\
\hline Notch1 & $\begin{array}{l}\text { (F) 5'-CCGCAGTTGTGCTCCTGAA-3' } \\
\text { (R) 5'-ACCTTGGCGGTCTCGTAGCT-3' }\end{array}$ & 109 \\
\hline$\beta$-catenin & $\begin{array}{l}\text { (F) 5'-CCTTTGTCCCGCAAATCATG-3' } \\
\text { (R) 5'-ACGTACGGCGCTGGGTATC-3' }\end{array}$ & 101 \\
\hline$C X C R 4$ & $\begin{array}{l}\text { (F) 5'-CAGTGGCCGACCTCCTCTT-3' } \\
\text { (R) 5'-ACATGGACTGCCTTGCATAGG-3' }\end{array}$ & 100 \\
\hline SOX2 & $\begin{array}{l}\text { (F) 5'-TGCGAGCGCTGCACAT-3' } \\
\text { (R) 5'-CGGGCAGCGTGTACTTATCC-3' }\end{array}$ & 96 \\
\hline$A L D H 3 A 1$ & $\begin{array}{l}\text { (F) 5'-TCCAGCAACGACAAGGTGATT-3' } \\
\text { (R) 5'-GGCAGAGAGTGCAAGGTGATG-3' }\end{array}$ & 101 \\
\hline
\end{tabular}

the lower chamber in DMEM/F12 medium containing $10 \mathrm{ng} / \mathrm{ml}$ EGF, $5 \mathrm{ng} / \mathrm{ml} \mathrm{bFGF,} 2.5 \mu \mathrm{g} / \mathrm{ml}$ insulin, and $2 \mu \mathrm{l} / \mathrm{ml} 50 \mathrm{X}$ B27. By Day 4 , mammospheres were observed by microscopy, the mammosphere-forming efficiency (MFE) was counted (diameter of mammospheres $>60 \mu \mathrm{M}$ ) and gene expression was detected by quantitative real-time PCR (qRT-PCR).

$q R T-P C R$. Total-RNA from the single-cell suspension was extracted using TRIzol Reagent according to the manufacturer's instructions, and the RNA was then stored at $-80^{\circ} \mathrm{C}$ until use. The RNA from each sample was reverse transcribed using random primers and MMLV. Real-time PCR was performed on cDNA samples using a SYBR-Green Master Mix (Applied Biosystems, Carlsbad, CA). The sequences of the gene-specific primers and the lengths of the PCR products are listed in Table I; PCR primers were designed using Primer Express Software, version 2. The reactions were conducted using the ABI PRISM 7900HT (Applied Biosystems) according the following conditions: $50^{\circ} \mathrm{C}$ for $2 \mathrm{~min}, 95^{\circ} \mathrm{C}$ for $10 \mathrm{~min}$ and 40 cycles of $95^{\circ} \mathrm{C}$ for $15 \mathrm{sec}$ and $60^{\circ} \mathrm{C}$ for $60 \mathrm{sec}$. The data were normalized to the reference gene GAPDH, and gene-specific amplification was confirmed by determining the melting curves of the PCR products.

Statistical analysis. The results are presented as the mean \pm SD for at least three individual experiments for each group. The statistical analysis was performed using SPSS 16.0 statistical Software and GraphPad Prism 4.0 software (San Diego, CA). Statistical differences were determined using either the two-tailed Student's t-test or the one-way analysis of variance (ANOVA). The level of statistical significance was defined as $\mathrm{P} \leq 0.05$

\section{Results}

Mammospheres enrich breast CSCs. Single MCF-7 cells were cultured in suspension $\left(2 \times 10^{4}\right.$ cells $\left./ \mathrm{ml}\right)$, as described previously, to produce mammospheres. In general, apparent mammospheres could be observed by microscopy by Day 6 (Fig. 1A and B). The shape of the mammospheres was round, with relatively regular morphology and an average diameter of $160 \mu \mathrm{m}$. The mammosphere formation efficacy (MFE) was about 180/10,000 MCF-7 cells, and the cells in the mammospheres showed a higher colony formation ability in vitro and higher tumorigenicity in vivo than whole MCF-7 cell populations, suggesting that the cell population was enriched with breast cancer stem/ progenitor cells in agreement with a previous report (9).

Flow cytometry was then performed to assess the percentage of $\mathrm{CD} 44^{+} / \mathrm{CD} 24^{\text {low/- }}$ cells in mammosphere cultures and MCF-7 monolayer cultures. The cells in the upper left quadrant were classified as $\mathrm{CD} 44^{+} / \mathrm{CD} 24^{\text {low/- }}$ cells (Fig. 1C). Our analysis indicated that, compared with a $2.1 \%$ positive expression in the MCF-7 monolayer culture cells, the expression of $\mathrm{CD}_{4} 4^{+}$ CD24 ${ }^{\text {low/ }}$ in MCF-7 mammosphere cells was significantly elevated to $10.4 \%(\mathrm{P}<0.01)$. These results are consistent with previous reports, in which the percentage of $\mathrm{CD} 44^{+} / \mathrm{CD} 24^{\text {low/- }}$ cells in mammosphere populations ranged from $10-12.1 \%$, while in MCF-7 monolayer culture cells the percentage was from $1.6-2.1 \%(11,12)$.

We performed qRT-PCR analysis to further determine the expression of Wnt1, Notch1, $\beta$-catenin, CXCR4, SOX2 and ALDH3A1 in mammosphere cells and MCF-7 monolayer culture cells. The results indicated that mammosphere cells showed significantly $(\mathrm{P}<0.01)$ increased expression of Wntl [fold change (FC), 2.25], Notchl (FC, 2.45), $\beta$-catenin (FC, 1.72), CXCR4 (FC, 4.68), SOX2 (FC, 4.25) and ALDH3A1 (FC, 5.38) compared with MCF-7 monolayer culture cells (Fig. 1D).

Identification of normal mammary and breast cancer fibroblasts. Fibroblasts were isolated and cultured as described previously. After culturing for 1-2 weeks, a mixture of both fibroblast and epithelial cells was observed under inverted microscopy (Fig. 2A). In light of the lower tolerance to trypsin 

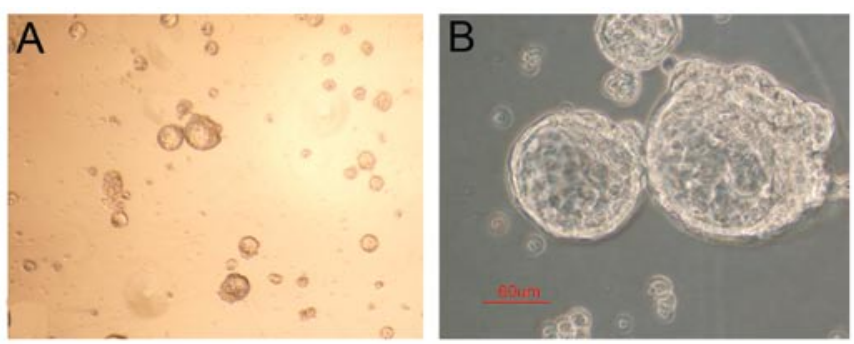

C
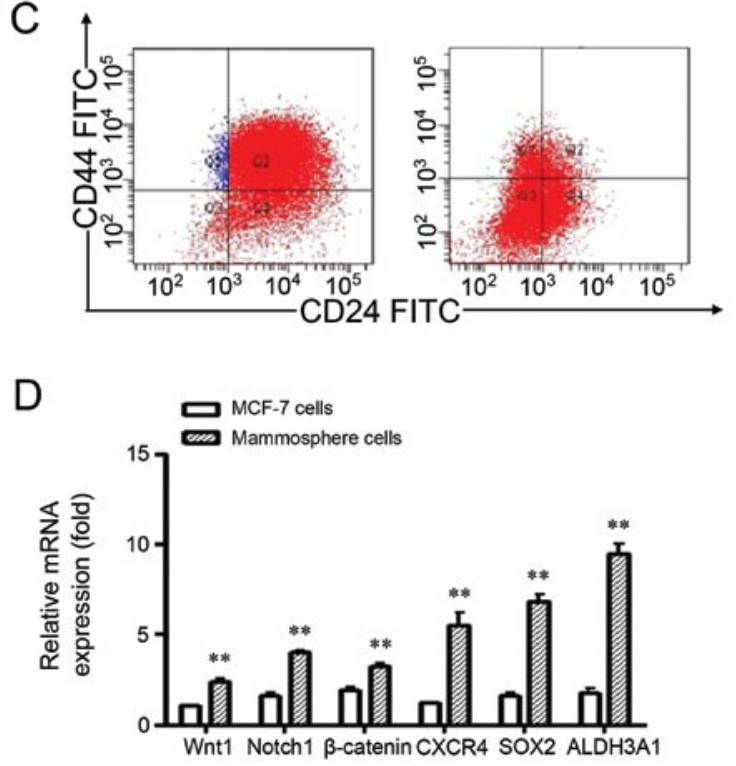

Figure 1. Mammosphere culture of MCF-7 cells. (A) Mammosphere formation of MCF-7 cells (x4). (B) Mammosphere formation of MCF-7 cells (x20). (C) CD44 and CD24 expression in MCF-7 monolayer cells (left) and mammospheres (right) as detected by fluorescence activated cell-sorting (FACS). (D) Real-time PCR analysis of the gene expression of Wnt1, Notch1, $\beta$-catenin, $C X C R 4, S O X 2$ and $A L D H 3 A 1$.

that fibroblasts have compared to epithelial cells (stromal cells are more easily detached by trypsin), we cultured and repeatedly digested these cells to separate and purify fibroblasts. After two or three subcultured generations, pure fibroblasts populations were achieved; the cells had a uniform appearance of a long fusiform shape typical of fibroblastic morphology when viewed by inverted microscopy (Fig. 2B).

Fibroblasts are normally defined by the concurrent expression of the smooth muscle marker $\alpha$-smooth muscle actin ( $\alpha$-SMA), the mesenchymal marker vimentin and FSP; on the other hand, CK (cytokeratin) was confirmed as an endothelial cell marker. To identify whether cells obtained by the above method were fibroblasts, the cells were stained with antibodies against $\alpha$-SMA, vimentin, FSP and CK by immunohistochemistry. Vimentin, $\alpha$-SMA, and FSP but not $C K$, were expressed in these cells, indicating that the cells we collected through this method were fibroblasts (Fig. 2C).

Effect of normal mammary and breast cancer fibroblasts on MCF-7 mammosphere formation. To determine the influence of fibroblasts on MCF-7 cell migration and mammosphere formation, MCF-7 cells were co-cultured with NMFs or BCFs in a transwell system (Fig. 3A). Mammospheres was observed
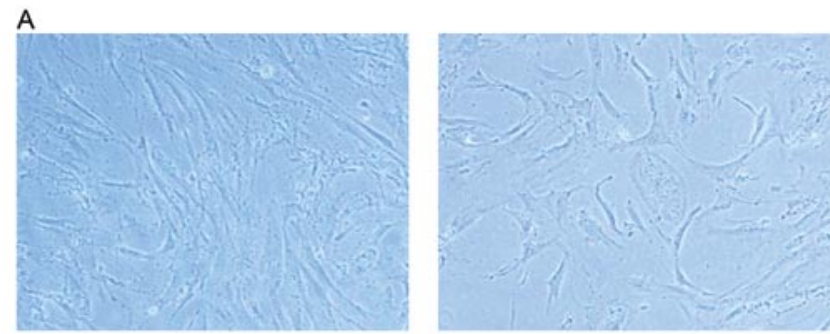

B
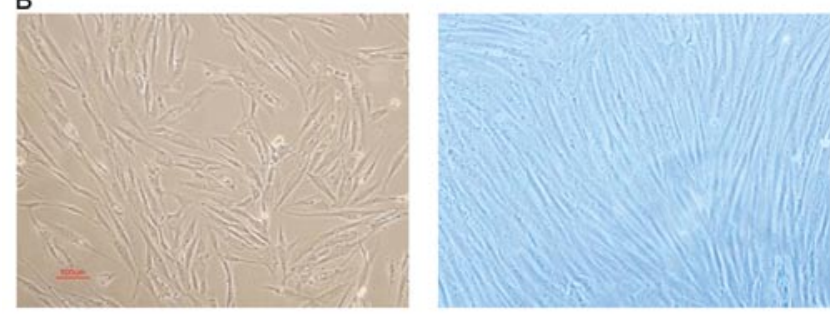

C
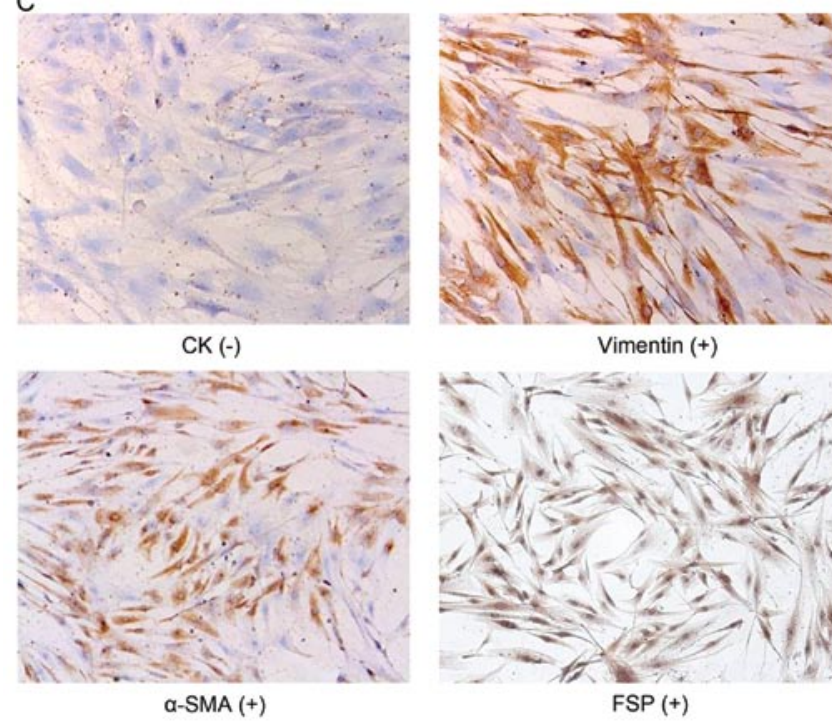

Figure 2. Identification of fibroblasts. (A) Admixture of fibroblasts and epithelial cells (x100). (B) Fibroblast cells are morphologically characterized as large spindle-shaped cells with indented nuclei (x100). (C) Immunohistochemistry for $\mathrm{CK}(-)$, Vimentin(+), $\alpha$-SMA(+), FSP(+) (x100).

by microscopy daily, and the MFE in each group was calculated on Day 7.

We found that BCFs promoted the migration of MCF-7 cells in the transwell culture system. When BCFs were co-cultured with MCF-7 under mammosphere culture conditions, the time of mammosphere formation was shorter, the volumes of the mammospheres were greater and the MFE was higher than both NMFs and the control group. Mammosphere formation in the BCFs group was enhanced 3.2-fold over control cultures. NMFs showed an approximately 1.3-fold greater MFE compared with the control group, whereas BCFs showed an approximately 2.5-fold increase over the NMFs group. Our study demonstrates that fibroblasts, especially those in cancer tissues, increase the mammosphere formation efficiency of MCF-7 cells in our co-culture system, reflecting their ability to increase the clonogenic efficiency of breast cancer cells (Fig. 3B-D).

Regulation of mammosphere signaling proteins by breast cancer and normal mammary fibroblasts. To further determine the 
A
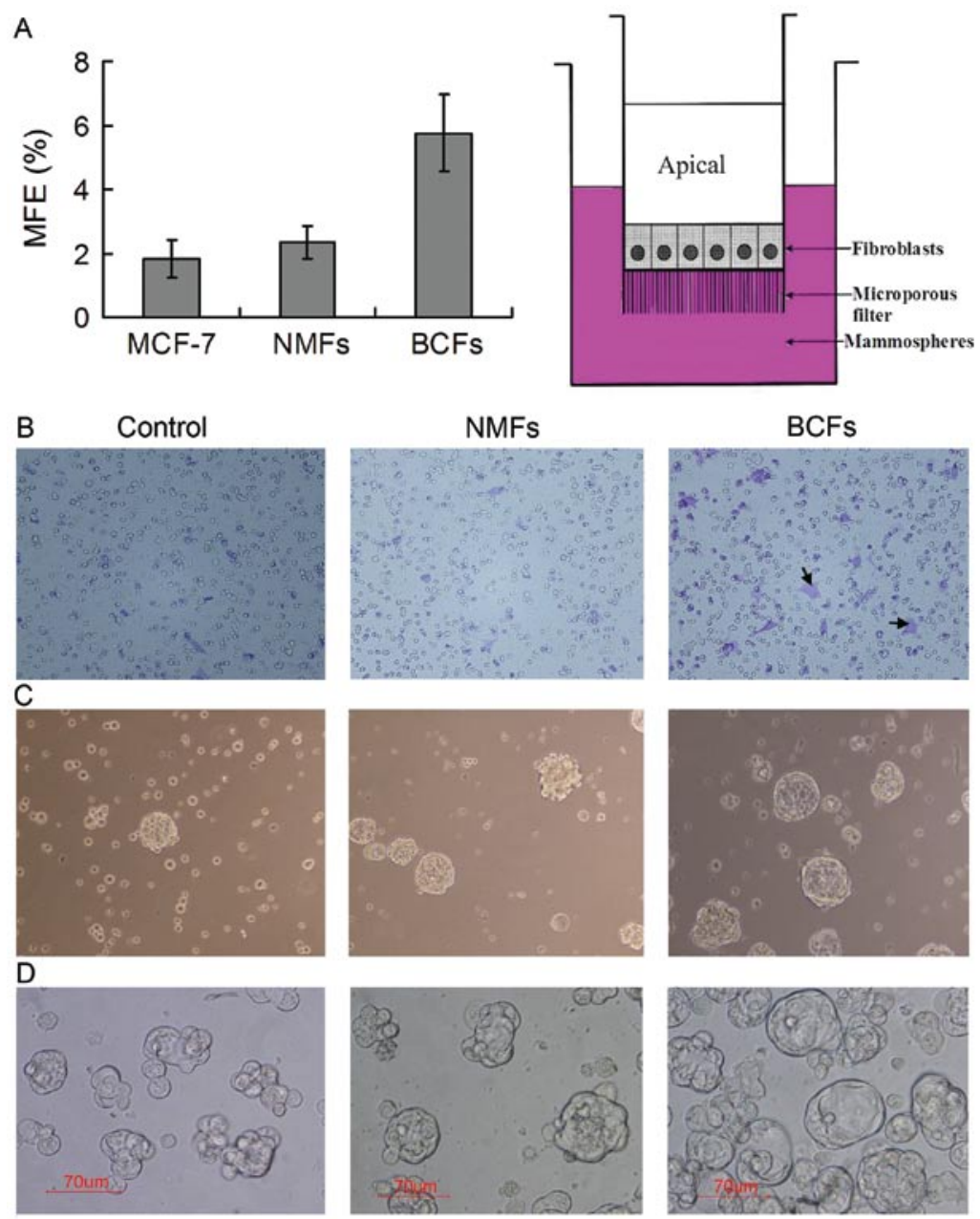

Figure 3. Effect of breast cancer (BCFs) and normal mammary fibroblasts (NMFs) on MCF-7 cell migration and mammosphere formation. (A) Comparison of mammosphere-forming efficiency, NMFs, BCFs. (B) Migration of MCF-7 cells; the arrow points to the migrating MCF-7 cells. (C) MCF-7 cells co-cultured with NMFs, Day 2. (D) MCF-7 cells co-cultured with BCFs, Day 7.
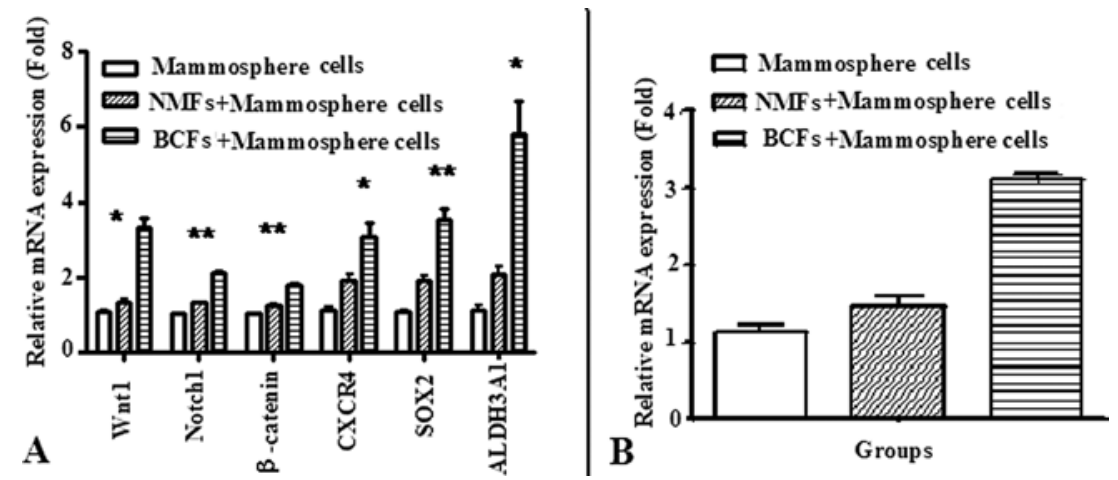

Figure 4. Effect of breast cancer (BCFs) and normal mammary fibroblasts (NMFs) on stem cell-related gene expression. NMFs and BCFs were co-cultured with mammospheres.

impact of breast cancer and normal mammary fibroblastls on several major mammosphere signaling proteins, we examined the differential gene expression by qRT-PCR. The analysis of gene expression profiling showed that the expression levels of Wnt 1 in MCF-7 mammospheres were significantly up-regulated by 3.18 -fold in the BCFs co-culture group and by 1.27 -fold in the NMFs co-culture group over the control group (Fig. 4).
Similar trends were also observed for the expression of other signaling proteins, such as $\beta$-catenin, Notch1, CXCR4, SOX2 and $A L D H 3 A 1$, with either NMFs or BCFs, as follows: Wnt 1 , FC of 1.27 and 3.18, respectively; $\beta$-catenin, FC of 1.22 and 1.75 , respectively; Notchl, FC of 1.31 and 2.09, respectively; CXCR4, FC of 1.73 and 2.77, respectively; SOX2, FC of 1.8 and 2.77, respectively; and $A L D H 3 A 1, \mathrm{FC}$ of 1.85 and 5.23, respectively. 


\section{Discussion}

Stem cells are characterized by their ability to self-renew as well as generate differentiated cells within each organ. Numerous studies have provided strong evidence for the existence of mammary stem cells capable of self-renewal and differentiation into the basal and luminal lineages comprising the functional mammary epithelium (13). Recently, Sheridan et al (14) reported the existence of a $\mathrm{CD} 44^{+} / \mathrm{CD} 24^{\text {low/ }}$ subpopulation in breast cancer cell lines that possess more invasive and proliferative properties than other breast cancer cell populations. Patrawala et al (6) confirmed that the $\mathrm{CD} 44^{+} / \mathrm{CD} 24^{\text {low } /}$ subpopulation of cancer cells have BCSC properties, and as compared to unsorted cells, only a low number of $\mathrm{CD} 44^{+} /$ $\mathrm{CD} 24^{\text {low/ }}$ cells is sufficient for tumor initiation.

During breast carcinogenesis, concurrent morphological and molecular changes occur in stromal and epithelial compartments, which form a microenvironment suitable for tumor growth. The tumor microenvironment is composed of mesenchymal cells, extracellular matrix components, vascular endothelial cells and inflammatory cells; fibroblasts (also termed myofibroblasts or cancer-associated fibroblasts) represent the majority of the stromal cells. The cells are able to modify the epithelial cell phenotype by direct cell-to-cell contacts, through soluble factors or by modification of ECM components. At the same time, breast cancer cells promote MMP-2 and MMP-9 expression in cancer-associated fibroblasts by stromalepithelial interactions, which form a microenvironment that contributes to the invasion and metastasis of malignant tumors (15). Premalignant mammary epithelial cells exposed to senescent human fibroblasts were reported to irreversibly become invasive and undergo a full malignant transformation (16). Cancer cells were shown to be suppressed by young and quickly proliferating non-cancer cells, such as embryonic stem cells (17). These reports demonstrate that stromal alterations accompany or even precede the malignant conversion of epithelial cells.

In a previous study, we described an in vitro culture system to propagate primary mammary epithelial stem cells in an undifferentiated state based on their ability to proliferate as spherical structures (mammospheres) in the presence of growth factors, and that these cultured mammospheres exhibited BCSC properties (9). In this study, the mammosphere formation efficacy was approximately 180/10,000 MCF-7 cells. Flow cytometry (FCM) analysis showed that, compared with $2.1 \%$ expression in the MCF-7 monolayer culture cells, the expression of $\mathrm{CD} 44^{+} / \mathrm{CD} 24^{\text {low/ }}$ in MCF-7 mammosphere cells was significantly elevated $(10.4 \%)(\mathrm{P}<0.01)$. The proportion of cells with the $\mathrm{CD} 44^{+} / \mathrm{CD} 24^{\text {low/ }}$ phenotype in the mammosphere subpopulation was nearly 5 -fold higher than that in the general MCF-7 population, suggesting that, consistent with several reports, mammosphere cultivation enriches CSCs $(18,19)$.

Studies on normal breast stem cells and BCSCs have revealed that several key signaling pathways are involved in the self-renewal and maintenance of the stem cell pool, including the Wnt/ $\beta$-catenin, Notch, Hedgehog $(\mathrm{Hh})$, transforming growth factor (TGF)- $\beta$, PTEN and Bmi signaling pathways (20-22). The Wnt pathway is involved in the regulation of self-renewal in normal breast stem cells and causes an increase in mammary stem cells (23), and the expression of
Wnt1 and $\beta$-catenin increases in BCSCs compared to non-BCSCs (24). Notch signaling promotes the self-renewal of mammary stem cells as well as the proliferation of early progenitor cells, and unregulated Notch signaling prevents the terminal differentiation of mammary epithelial cells (25). Hh signaling components, such as PTCH1, Gli1 and Gli2 are highly expressed in normal human mammary stem/progenitor cells (26). Consistent with previous results, we observed an enhanced expression of Wnt1, Notch1, $\beta$-catenin, CXCR4, SOX 2 and ALDH3A1 in mammosphere cells as compared with MCF-7 monolayer culture cells.

Furthermore, Kucia et al (27) found that CSCs exhibit the same metastasis mechanisms as normal stem cells, and that transplantation and the SDF-1/CXCR4 axis play an important part in these events. In our study, cancer mammospheres strongly expressed CXCR4, which suggests that CXCR4 may be involved in regulating breast cancer stem cell metastasis and invasion. SOX2 is a transcription factor essential for the self-renewal and differentiation of embryonic $(28,29)$ and neural stem cells (30). Eriksson et al (31) found that $\mathrm{CD} 44^{+} \mathrm{CD} 24^{\text {low }}-$ cells isolated from breast tumor patient pleural effusions expressed SOX2. ALDH3A1 is a NAD-dependent enzyme responsible for the oxidation of intracellular aldehydes. A recent study demonstrated that ALDH3A1 not only inhibits cell proliferation by affecting the MAPK pathway but also converts the activated drug to the inactive metabolite (32), and Ginestier et al (33) discovered that ALDH1 expression in breast carcinomas correlated with poor prognosis. Here, we found that the expression of CXCR4, SOX2 and ALDH3A1 were 4.68-, 4.25- and 5.38-fold higher, respectively, in mammosphere cells than in monolayer cells.

We further examined whether cancer-associated fibroblasts could influence MCF-7 cell mammosphere formation. Using the transwell co-culture system, we observed that cancerassociated fibroblasts significantly enhanced the formation of MCF-7 cell mammospheres, as mammosphere formation in the breast cancer fibroblast group was enhanced 3.2-fold over control cultures. Our study shows that fibroblasts, especially cancer fibroblasts, increase the mammosphere formation efficiency of MCF-7 cells in a co-culture system, reflecting their ability to increase the clonogenic efficiency of this cell line. NMFs increased the MCF-7 MFE approximately 1.3-fold compared with the control group, while BCFs caused an approximately 2.5 -fold increase compared with the NMFs group. Our data indicates that tumor fibroblasts may provide a functional microenvironment to play an important role in mammosphere formation.

The study also revealed that fibroblasts have a broad effect on signaling protein expression; Wnt1 expression levels were significantly up-regulated 3.18 -fold in the BCFs co-culture group over the control group, whereas the NMFs co-culture group caused a 1.27 -fold increase over the control group. Similar trends were also observed for the expression of other signaling proteins.

In general, these studies show that the microenvironment provides a suitable condition for cancer cells by secreting cytokines, which are associated with CSC growth and differentiation. These results indicate that the role of cancer microenvironments cannot be ignored, and that they also provide a reference for clinical and fundamental research. 
Cells exist in a complicated ecological system and make interactions by juxtacrine and paracrine signaling, and any form of inappropriate signaling can be carcinogenic. The approach of suppressing tumor growth by blocking specific signal transmission will be a breakthrough for breast cancer therapy. Our study suggests that cancer-associated fibroblasts may represent a new target for cancer therapy by blocking specific signaling molecules and downstream effectors to sacrifice malignant cells. These results may also be useful in finding new therapies for CSC eradication.

In summary, cancer fibroblast cells can promote the MFE and up-regulate stem cell-related gene expression in breast cancer cells, indicating that the cancer microenvironment provides a suitable condition for CSC maintenance.

\section{Acknowledgements}

The study was supported by the Leading Academic Discipline Project of the Shanghai Municipal Education Committee (J50208), the National Natural Science Foundation of China (30670798) and the Suzhou Planning Project of Science and Technology (SYS201062).

\section{References}

1. Singh SK, Hawkins C, Clarke ID, et al: Identification of human brain tumour initiating cells. Nature 432: 396-401, 2004.

2. Al-Hajj M, Wicha MS, Benito-Hernandez A, et al: Prospective identification of tumorigenic breast cancer cells. Proc Natl Acad Sci USA 100: 3983-3988, 2003.

3. Haraguchi N, Utsunomiya $\mathrm{T}$, Inoue $\mathrm{H}$, et al: Characterization of a side population of cancer cells from human gastrointestinal system. Stem Cells 24: 506-513, 2006.

4. Bonnet D and Dick JE: Human acute myeloid leukemia is organized as a hierarchy that originates from a primitive hematopoietic cell. Nat Med 3: 730-737, 1997.

5. Goodell MA, McKinney-Freeman S and Camargo FD: Isolation and characterization of side population cells. Methods Mol Biol 290: 343-352, 2005.

6. Patrawala L, Calhoun T, Schneider-Broussard R, et al: Side population is enriched in tumorigenic, stem-like cancer cells, whereas $\mathrm{ABCG}^{+}$and $\mathrm{ABCG} 2^{-}$cancer cells are similarly tumorigenic. Cancer Res 65: 6207-6219, 2005.

7. Xu JX, Morii E, Liu Y, et al: High tolerance to apoptotic stimuli induced by serum depletion and ceramide in side-population cells: high expression of CD55 as a novel character for sidepopulation. Exp Cell Res 313: 1877-1885, 2007.

8. Cariati M, Naderi A, Brown JP, et al: Alpha-6 integrin is necessary for the tumourigenicity of a stem cell-like subpopulation within the MCF7 breast cancer cell line. Int J Cancer 122: 298-304, 2008.

9. Lin SC, Huang MZ, Zhang FC and Zhang YY: Enrichment of breast cancer stem cells and correlative gene expression. J Shanghai Jiaotong Univ (Medical Sci) 28: 2008.

10. Chepko G and Dickson RB: Ultrastructure of the putative stem cell niche in rat mammary epithelium. Tissue Cell 35: 83-93, 2003.

11. Phillips TM, McBride WH, Pajonk F: The response of CD24/-low/ $\mathrm{CD} 44^{+}$breast cancer-initiating cells to radiation. J Natl Cancer Inst 98: 1777-1785, 2006

12. Huang MZ, Zhang FC and Zhang YY: Influence factors on the formation of mammospheres from breast cancer stem cells. Beijing Da Xue Xue Bao 40: 500-504, 2008 (In Chinese).
13. Visvader JE and Lindeman GJ: Mammary stem cells and mammopoiesis. Cancer Res 66: 9798-9801, 2006.

14. Sheridan C, Kishimoto H, Fuchs RK, et al: CD44 ${ }^{+}$CD24- breast cancer cells exhibit enhanced invasive properties: an early step necessary for metastasis. Breast Cancer Res 8: R59, 2006.

15. Singer CF, Kronsteiner N, Marton E, et al: MMP-2 and MMP-9 expression in breast cancer-derived human fibroblasts is differentially regulated by stromal-epithelial interactions. Breast Cancer Res Treat 72: 69-77, 2002.

16. Parrinello S, Coppe JP, Krtolica A and Campisi J: Stromal-epithelial interactions in aging and cancer: senescent fibroblasts alter epithelial cell differentiation. J Cell Sci 118: 485-496, 2005.

17. Ukraintseva SV and Yashin AI: Treating cancer with embryonic stem cells: rationale comes from aging studies. Front Biosci 10: 588-595, 2005

18. Dontu G, Abdallah WM, Foley JM, et al: In vitro propagation and transcriptional profiling of human mammary stem/progenitor cells. Genes Dev 17: 1253-1270, 2003.

19. Yuan X, Curtin J, Xiong Y, et al: Isolation of cancer stem cells from adult glioblastoma multiforme. Oncogene 23: 9392-9400, 2004.

20. Zhou J, Wulfkuhle J, Zhang H, et al: Activation of the PTEN/ mTOR/STAT3 pathway in breast cancer stem-like cells is required for viability and maintenance. Proc Natl Acad Sci USA 104: 16158-16163, 2007.

21. Brennan KR and Brown AM: Wnt proteins in mammary development and cancer. J Mammary Gland Biol Neoplasia 9: 119-131, 2004.

22. Boulanger CA, Wagner KU and Smith GH: Parity-induced mouse mammary epithelial cells are pluripotent, self-renewing and sensitive to TGF-betal expression. Oncogene 24: 552-560, 2005.

23. Lindvall C, Evans NC, Zylstra CR, et al: The Wnt signaling receptor Lrp5 is required for mammary ductal stem cell activity and Wnt1-induced tumori-genesis. J Biol Chem 281: 35081-35087, 2006.

24. Kruger JA, Kaplan CD, Luo Y, et al: Characterization of stem cell-like cancer cells in immune-competent mice. Blood 108: 3906-3912, 2006

25. Dontu G, Jackson KW, McNicholas E, et al: Role of Notch signaling in cell-fate determination of human mammary stem/ progenitor cells. Breast Cancer Res 6: R605-R615, 2004.

26. Liu S, Dontu G, Mantle ID, et al: Hedgehog signaling and Bmi-1 regulate self-renewal of normal and malignant human mammary stem cells. Cancer Res 66: 6063-6071, 2006.

27. Kucia M, Reca R, Miekus K, et al: Trafficking of normal stem cells and metastasis of cancer stem cells involve similar mechanisms: pivotal role of the SDF-1-CXCR4 axis. Stem Cells 23: 879-894, 2005.

28. Park IH, Zhao R, West JA, et al: Reprogramming of human somatic cells to pluripotency with defined factors. Nature 451: 141-146, 2008.

29. Kopp JL, Ormsbee BD, Desler M and Rizzino A: Small increases in the level of Sox 2 trigger the differentiation of mouse embryonic stem cells. Stem Cells 26: 903-911, 2008.

30. Cavallaro M, Mariani J, Lancini C, et al: Impaired generation of mature neurons by neural stem cells from hypomorphic Sox2 mutants. Development 135: 541-557, 2008.

31. Eriksson M, Guse K, Bauerschmitz G, et al: Oncolytic adenoviruses kill breast cancer initiating $\mathrm{CD} 44^{+} \mathrm{CD} 24^{-/ \mathrm{low}}$ cells. Mol Ther 15 : 2088-2093, 2007.

32. Muzio G, Trombetta A, Martinasso G, et al: Antisense oligonucleotides against aldehyde dehydrogenase 3 inhibit hepatoma cell proliferation by affecting MAP kinases. Chem Biol Interact 143-144: 37-43, 2003.

33. Ginestier C, Hur MH, Charafe-Jauffret E, et al: ALDH1 is a marker of normal and malignant human mammary stem cells and a predictor of poor clinical outcome. Cell Stem Cell 1: 555-567, 2007. 